\title{
Diversion Radiometer Data for Water Vapor Direction Delay
}

\author{
Ahmad Khalf Aldhafari
}

\begin{abstract}
The radiometers for probing water vapor, temperature and cloud liquid water do not show the high accuracy and stability which is needed for some applications like turbulent weather conditions. The Form of direction delay from atmospheric water vapor is a main error source in geodetic measurements made with VLBI. A dual channel water vapor radiometer, operating near the $22 \mathrm{GHz}$ water vapor line, and measuring water vapor-induced delay with good conditions. Theory shows that water vapor direction delay $\Delta \mathrm{LV}$ is related to a linear combination of saturationcorrected sky brightness temperatures, measured the water vapor line. Sky brightness temperatures are saturationcorrected or "linearized" using estimates of effective sky temperature made from surface temperature. A systematic method for reducing dual-frequency water vapor radiometer data to water vapor path delay has been developed Coefficients are found from a regression of theoretical brightness temperatures versus radiosonde measured delay. Regression solutions are constrained to remove liquid water contributions and to give the correct slope for radiometer versus radiosonde path delay.
\end{abstract}

Index Terms - Water vapor path, atmospheric water vapor, spacecraft.

\section{INTRODUCTION}

An Advanced Water Vapor Radiometer (AWVR) is presently being developed at JPL to support planned radio science experiments involving NASA's Deep Space Network (DSN) and the Cassini spacecraft, The AWVR will operate at frequencies from 22 to $31 \mathrm{GHz}$, and will be used to measure atmospheric water vapor along a line of sight from ground based DSN antennas to the spacecraft. The water vapor data will then be used in conjunction with other atmospheric parameters A well established technique to retrieve altitude profiles of water vapor concentration in the middle atmosphere is by microwave radiometry.

Water vapor is variable delay to radio signals passing through the earth's troposphere that can limit the phase stability and coherence time for VLBI or for involve precise spacecraft trackin and have potential accuracies at the few centimeter level and constitute a limiting error source, Water vapor induced direction delay can vary from less than I to more than 30 centimeters depending on climate and weather along a line of sight with a water vapor radiometer (WVR)[1]., and the direction delay can be solved for as part of the final fitting of residuals in the VLBI data reduction process and inferred from these data using models and estimates of the atmospheric temperature and pressure. A microwave radiometer will operate day or night and under

Manuscript received October 20, 2012; revised November 27, 2012

A. K. Aldhafari is with School of Electronics Engineering and Information, Beihang University, China (e-mail: sabocah@hotmail.com) all weather conditions except rain or heavy snow.this paper is to present techniques for conversion of radiometer data to water vapor path delay.

\section{MEASUREMENT WATER VAPOR RADIOMETER}

The radiometer used in this experiment is a three channel microwave instrument operating at frequencies around the water vapor spectral line centered at $22 \mathrm{GHz}$. The basic design of the instrument is described by jassen (1985). The addition of a hot load to the system has greatly improved the system calibration and allows better gain stability during periods when the meteorological conditions are not suitable for calibration. the instrument is fully steerable in both azimuth and elevation, allowing full sky coverage to be obtained.

The water vapor spectral line at $22 \mathrm{GHz}$ is rather weak, which implies that radiation emitted by water vapor molecules at high altitudes will be only slightly attenuated when it arrives at the surface of earth, for all realistic densities of atmospheric water vapor. A complication is that liquid water also emits radiation of comparable or greater intensities than the vapor at the frequencies $(22 \mathrm{GHz})$. However, the two radiation processes exhibit distinctly different spectral features [2], and the liquid contribution can be separated from the vapor contribution by measuring the emission at two different frequencies. Although the instrument takes measurements at three frequencies $(20,22$ , and $31 \mathrm{GHz})$, only measurements at two frequencies (20 and $31 \mathrm{GHz}$ ) are actually used in the calculation of integrated perceptible water vapor with the higher frequency contributing most to the estimate of the liquid water component. The choice of frequencies has been discussed by numerous authors.

The addition of measurement from the third frequency channel at $22 \mathrm{GHz}$ may produce a slight $(<7 \%)$ improvement in rms performance over thr two-frequency approach. The lower frequency used in the calculation of the perceptible water vapor $(20 \mathrm{GHz})$ is positioned close to the center of water vapor emission line, while the other frequency $(31 \mathrm{GHz})$ is placed well away from the line center. Measurement at this frequency $(20 \mathrm{GHz})$ are better correlated with the integrated water vapor than are measurement at the line center.

Figure 1 shows a block diagram of a typical total power radiometer, which consists of an antenna, a low-noise amplifier (LNA), an RF filter, a mixer to down-convert the incoming signal to the intermediate frequency (IF), an IF amplifier ,an IF filter (whose bandwidth B typically determines the bandwidth of the receiver), a square-law detector (whose output voltage is proportional to its input power), and an integrator. 




Fig. 1. Block diagram of total power radiometer

\section{TECHNICAL OVERVIEW}

The intensity and phase delay of satellite transmissions depend on atmospheric fluctuations as well as technical factors such as orbit instabilities of the spacecraft or thermally driven antenna distortions.

A solution of the radiative transfer equation governing propagation of microwave radiation downward through a non scattering atmosphere in thermodynamic equilibrium is[3].

$$
T_{B}=T_{c} e^{-\tau(\infty)}+\int_{0}^{\infty} T \alpha e^{-\tau(s)} d s
$$

The optical depth, a is the absorption coefficient (distance-1), $\mathrm{s}$ is distance upward along the signal path, $\mathrm{T}$ is physical temperature of the air, TB is the sky brightness temperature, and TC is the cosmic blackbody temperature of about $2.8 \mathrm{~K}$. Typical half-power antenna beam widths of 5 to 10 degrees are large enough that all celestial sources of radiation are negligible except for the sun water vapor, oxygen, and the liquid water droplets in clouds or fog. Water vapor has a spectral line at $22 \mathrm{GHZ}$. Sensitivity to water vapor is achieved by choosing a primary frequency on or near this line, The oxygen contribution is from a complex of spectral lines near $60 \mathrm{GHz}$ and has a frequency dependence which is also approximately f2. About 82 percent of the oxygen contribution is removed by the $\mathrm{f} 2$ dependence assumed for liquid emission, we recognize that a very important consideration is that a frequency pair be chosen which minimizes variation with altitude of the weighting function $\mathrm{W}^{\prime}$ (s) (Ref. 3):

$$
W^{\prime}(s)=\frac{T\left(T-T_{c}\right)}{\rho}\left[\frac{\alpha_{v, 1}}{f_{1}^{2}}-\frac{\alpha_{v, 2}}{f_{2}^{2}}\right]
$$

where $\alpha \mathrm{V}$ is the absorption coefficient for water vapor and $\rho$ is water vapor density. The penalty for a nonconstant Eq. (2) along the observing path is appreciable error whenever the water vapor altitude distribution varies markedly from some calibration distribution. .

\section{TECHNIQUE OUTLINE}

The system here can developed into part . the first part for converting raw radiometer data and surface temperature into linearized sky brightness temperatures. An important element of this procedure is the use of tipping curves to provide an instrumental calibration , The second part is calculation of suitable coefficients for use in fmding path delay. Two methods will be discussed for calculating coefficients, both using a regression analysis. In each case the coefficients are adjusted for differing climatic conditio by measurements of surface temperature, pressure, and relative humidity. Presence of the exponential terms in Eq. (1) prevents sky brightness temperature from increasing linearly with increasing water vapor path delay. In the limit of small opacities, the relation is linear. In the limit of extremely high opacities, If the linearized brightness temperature $\mathrm{T}^{\prime} \mathrm{B}$ is defined by

$$
\begin{aligned}
T_{B}^{\prime} & =T_{c}[1-\tau(\infty)]+\int_{0}^{\infty} T \alpha d s \\
& =T_{c}+\int_{0}^{\infty}\left(T-T_{c}\right) \alpha d s
\end{aligned}
$$

Then linearized brightness temperature may be calculated from measured brightness temperature TB using the following relation derived from Eqs. (1) and (3) [4]:

$$
\begin{gathered}
T_{B}^{\prime}=T_{c}-\left(T_{e f f}^{\prime}-T_{c}\right) \ln \left(1-\frac{T_{B}-T_{c}}{T_{e f f}-T_{c}}\right) \\
T_{e f f}=T_{e f f}^{\prime}=k_{e} T
\end{gathered}
$$

where $T$ is absolute surface temperature, $\mathrm{T}^{\top} \mathrm{B}$, the linearized brightness temperature, is not very sensitive to Teff and T'eff Figure 1 shows the linearization correction T'B- TB as a function of TB for three different estimates of Teff $=\mathrm{T}$ 'eff The correction becomes a significant error source only at very high sky temperatures.



Fig. 2. Correction $T^{\prime}{ }_{B}-$ TB required to linearize brightness temperatures $T_{B}$ for three effective temperatures $\mathrm{T}_{\text {eff }}$ FLD when hydrostatic pressure was applied

T'B,1 and T'B,2 are derived from measured brightness temperatures at the two frequencies using Eq. (4). $\mathrm{W}^{\prime}(0)$ is the weighting function of Eq. (2) evaluated at the surface to adjust path delay for varying values of surface temperature, pressure, and water vapor density,

$\bar{T}$ and $\bar{P}$ are nominal surface values, but are best chosen to be representative of the calibrating or operating conditions Expressions for the water vapor absorption coefficient $\alpha \mathrm{v}$ can be found in waters [2] and West water [5]. 


\section{MEASUREMENT BRIGHTNESS TEMPERATURE}

Radiometer is an analog signal which varies linearly with signal power., every radiometer contains two reference loads at carefully measured physical temperatures to establish a brightness temperature calibration. If the sky brightness temperature is TB, the ambient or base load temperature TA , and the hot load temperature $\mathrm{TH}$ ' then the corresponding number of counts NB, NA , NH (from an analog to digital converter) are related to brightness temperature by

$$
\begin{gathered}
T_{B}=T_{A}+\left(T_{H}-T_{A}\right) \gamma \\
T_{B}=T_{A}+\left(T_{H}+\Delta T_{H}-T_{A}\right) \gamma
\end{gathered}
$$

In practice Eq. (6) is imperfect because of losses and reflections in the horn, waveguide, and switching system.

\section{TiPping CURVES AND THE Hot LOAD CORRECTION}

Tipping curves are performed by taking measurements of sky brightness temperature at several elevation angles. When a straight line is fitted to linearized brightness temperatures from Eqs. (7) and (4) as a function of air mass, the expected intercept at zero air mass is TC. Suppose the intercept has a different value $\mathrm{T}^{\prime} \mathrm{B}(0) . \Delta \mathrm{TH}$ must be adjusted to achieve the desired intercept. Figure 3 shows a straight line fit before (dashed) and after (solid) adjustment of $\triangle \mathrm{TH}$ (The two lines intersect off the graph at $\mathrm{T}^{\prime} \mathrm{B}=\mathrm{T}^{\prime} \mathrm{A}$.)

If the hot load correction is initially taken to be zero, then one can show that

$$
\begin{gathered}
\Delta T_{H}=\frac{\left[T_{c}-T_{B}^{\prime}(0)\right]\left[T_{H}-T_{A}\right]}{\left[T_{B}^{\prime}(0)-T_{A}\right]} \\
\Delta T_{B}=\Delta T_{H}\left(\frac{T_{B}-T_{A}}{T_{H}-T_{A}}\right)
\end{gathered}
$$

New sky brightness temperatures $T_{B}+\Delta T_{B}$ are linearized with Eq. (4) and fitted again to a straight line. The process is repeated 2 as many times as necessary to adjust $T^{\prime}{ }_{B}(0)$, the intercept, to within about $0.1 \mathrm{~K}$ of $\mathrm{T}_{\mathrm{C}}$. Normally two or three iterations are sufficient. We generally use three points at 1, 1.5, and 2 air masses (90-,42-, and 30-degree elevation angles) for tipping curves. Repeating the measurement at four points of the compass (different azimuths) provides redundancy in the data and averages over spatial gradients. Various problems with the measurement system can cause a poor tipping curve. Quality criteria for a tipping curve are the value of the linear correlation coefficient, the size of the hot load correction and its agreement with a "historical" estimate, and the agreement of the estimated zenith path delay with those derived from tipping curves at other azimuths. Experience with an instrument will show to what degree $\Delta T_{H}$ is constant for each channel. One can then estimate error incurred by using nominal values of $\Delta T_{H}$ to replace tipping curves. In principle, the most meaningful delay is found by measurements along the line-of-sight to the radio source being observed. For an automated system, a horizon mask for each site and a solar ephemeris are needed to avoid pointing the instrument toward contaminating radiation. Under dry conditions, pointing along the line-of-sight is not likely to give measurable improvement over extrapolated zenith values. In fact, error may be introduced by sidelobe pickup and poorer performance of the data reduction algorithm at low elevation angles. We expect pointing to improve VLBI results under very humid, cloudy conditions.

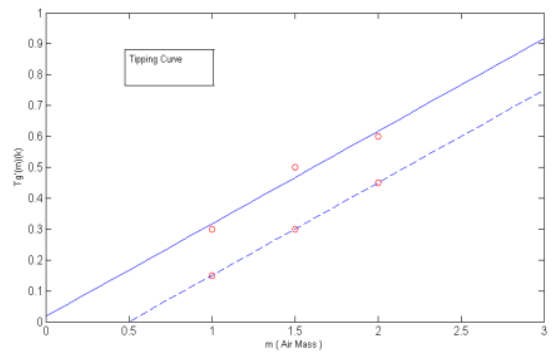

Fig. 3. Tipping curves with no hot load correction and with hot load correction required to give desired zero air mass intercept Tc hypothetical data points for each case are also shown

\section{CONCLUSION}

A systematic method for reducing dual-frequency water vapor radiometer data to water vapor path delay has been developed. Linearized sky brightness temperatures and path delay coefficients are computed in separate procedures and then combined to calculate path delay. Sky brightness temperatures may be measured by using either tipping curves directly or by using nominal hot load corrections based on tipping curves. Coefficients may be derived from brightness temperature data using a constrained regression analysis against radiosonde-measured path delays, or from a constrained regression analysis of theoretically calculated sky brightness temperatures against radiosonde measured path delays., pressure, and water vapor density, to allow use of the data reduction algorithm at any altitude and in any climate.

\section{REFERENCES}

[1] M. A. Janssen, "Atmospheric remote sensing by microwave radiometry," New York: Wiley \& sons, 1993.

[2] M. Tinto and J. W. Armstrong, "Spacecraft Doppler tracking as a narrow-band detector of gravitational radiation," Physical Review D., vo1.58, pp. 042002-1 -8, July 1998.

[3] D. L. Thacker et al., "Ground-based sensing of water vapor in the stratosphere and mesosphere," IEEE Trans. Instrum. Meas., vol. 44, no. 2, pp. 355-359, Apr. 1995.

[4] West water and E. R., "An Analysis of the Correction of Range Errors Due to Atmospheric Refraction by Microwave Radiometric Techniques," U.S. Department of Commercel ESSA Tech. Report, IER 30-ITSA 30, Boulder, CO, Mar. 1967.

[5] D. C. Hogg, F. 0. Guiraud, and M. T. Decker, "Measurement o f excess radio transmission length on earth-space paths," Astron. Astrophys, vol. 95, pp. 304-307, 1981.

Ahmad Khalf Aldhafari received the master degrees from beihang University. $\mathrm{He}$ is currently study for Ph.D. School of Electronics Engineering and Information at the beihang University His research interests are in radiometer. 